\title{
Valeur alimentaire des rations complètes à base d'ensilage d'herbe pour les vaches laitières
}

\author{
JP Dulphy 1, J Rouel 2, J Bony 2, JP Andrieu 1 \\ avec la collaboration technique de $\mathrm{M}$ Jailler \\ 1 INRA, station de recherches sur la nutrition des herbivores, 63122 Saint-Genès-Champanelle; \\ 2 Domaine d'Orcival, 63210 Rochefort-Montagne, France
}

(Reçu le 14 octobre 1992; accepté le 27 juillet 1993)

\begin{abstract}
Résumé - Trois essais (essais I, II et IV) ont été réalisés sur vaches laitières afin d'estimer, à travers les performances des animaux, la valeur alimentaire de rations complètes à base d'ensilage d'herbe, par rapport aux mêmes rations, le fourrage et le concentré étant distribués séparément. En moyenne, la distribution de la ration complète a fait augmenter les quantités de MS totales ingérées de $1,8 \mathrm{~kg}$, alors que l'apport de concentré était réduit de $0,5 \mathrm{~kg}$. Cette augmentation de quantité ingérée est due pour une bonne part au rehachage de l'ensilage dans la remorque mélangeuse (montrée par une baisse de la durée de mastication par $\mathrm{kg}$ de MS d'ensilage). Elle est due aussi probablement à la régularité de l'apport du concentré au cours de la journée. Le passage à la ration complète n'a en revanche pas modifié significativement la production de lait, qui a tout de même baissé en moyenne de $0,5 \mathrm{~kg} / \mathrm{j}$ pour le lait à $4 \%$ de $M G$, à cause d'une baisse également non significative de 1 point du taux butyreux. Les animaux ont repris $100 \mathrm{~g}$ de poids vif de plus/j avec la ration complète lorsque l'apport de concentré n'a pas été modifié (essais I et II). Compte tenu des observations effectuées, il apparaît que le mélange ensilage d'herbe + concentré a eu une valeur énergétique inférieure à celle des aliments distribués séparément. Un quatrième essai (III) avait pour objectif de mesurer l'effet du rehachage par la remorque mélangeuse et les conséquences de la diminution de la proportion de concentré dans une ration complète sur les durées journalières de mastication des rations.
\end{abstract}

ingestion / comportement alimentaire / ration complète / ensilage herbe / vache laitière

Summary - Feeding value of complete diets based on grass silage for dairy cows. Three trials (I, II and IV) were executed with dairy cows to determine, by means of animal performance, the feeding value of complete diets based on grass silage, compared with the same diets in which roughage and concentrate were fed separately. With complete diets the cows ate on average $1.8 \mathrm{~kg}$ more of total DM $(+2.3 \mathrm{~kg}$ of roughage, because the supply of concentrate was reduced experimentally by $0.5 \mathrm{~kg}$ ). This intake increment was mainly caused by the chopping of the silage in the mixing vessel. This was shown by a reduced chewing time per $\mathrm{kg} \mathrm{DM}$ of silage. The increased intake was probably also caused by the regularity of concentrate intake during the day. Compared with the classical diet, the complete diet did not significantly modify milk production, despite a decrease of $0.5 \mathrm{~kg}$ 
FCM/day caused by a non-significant decrease of $1 \mathrm{~g} / \mathrm{kg}$ in the milk fat content. The animals increased live-weight by $100 \mathrm{~g}$ per $d$ extra for the complete diet, when the supply of concentrate was not modified (trials I and II). Taking into account the observed results, it can be calculated that the energetic value of the complete diets is lower than that of the control diet, for the same proportion of concentrate. A further trial (III) was executed to measure the effect of chopping in the mixing vessel and the consequences of a reduction in the proportion of concentrate in the complete diet on chewing time for the diets.

\section{intake / feeding behaviour / complete diet / grass silage / dairy cow}

\section{INTRODUCTION}

Par rapport aux rations classiques, dont les constituants sont distribués séparément, les rations complètes sont obtenues en mélangeant le plus intimement possible les concentrés et les minéraux aux fourrages (Owen, 1971). De nombreux avantages ont été avancés (Hoden et Giger, 1984) pour ce type de ration : meilleure digestion des parois végétales, ingestion plus élevée, orientations fermentaires dans le rumen favorables à la production de lait et au taux butyreux, valorisation efficace de sous-produits industriels, pas de concentré en salle de traite, etc. Les progrès de la mécanisation et la recherche d'une simplification du rationnement ont enfin largement contribué à leur développement, davantage cependant en GrandeBretagne et aux États-Unis qu'en France.

II existe déjà des résultats conséquents avec cette technique (Bines, 1980 ; Sheehan et al, 1981; Lahr et al, 1983 ; Nocek et al, 1986). En outre, les revues bibliographiques de Rickaby (1978), Gill (1979), Bines (1980), Owen (1984), Hoden et Giger (1984) puis Sauvant et Giger (1984) font largement le point à ce sujet.

Les essais français ont porté principalement sur des rations à base d'ensilage de maïs (Augeard et al, 1986). C'est pourquoi nous avons entrepris une série d'essais sur celles à base d'ensilage d'herbe.

La plupart des essais montrent que les rations complètes sont mieux ingérées que les rations classiques, mais n'améliorent pas la production de lait (Hoden et Giger, 1984). C'est ce que nous avons voulu également vérifier en privilégiant l'étude du rôle d'une remorque mélangeuse équipée de couteaux. Un premier essai a donc été consacré à la comparaison d'une ration dont les composants (ensilage, concentré) étaient distribués séparément ou mélangés dans la remorque, à même niveau d'apport d'aliment concentré. Un second essai a été mis en place l'année suivante en tenant compte des résultats du premier, avec pour objectif de chiffrer la diminution possible d'aliment concentré consécutive à l'augmentation d'ingestion du fourrage de la ration complète. À ce second essai a été associé un troisième pour mesurer l'effet du rehachage dans la remorque et de la proportion de concentré dans la ration complète sur l'ingestion et les durées journalières de mastication des animaux. Enfin, dans un quatrième essai, la même comparaison que dans le premier a été effectuée en tentant de maintenir identiques les productions laitières entre les 2 lots comparés et en fixant une fois pour toutes le niveau d'apport journalier de concentré dans la ration complète. Les objectifs globaux de ces essais ont été très appliqués, sans mesure complémentaire au niveau de la digestion, mais en recherchant dans le troisième essai quelques explications aux résultats obtenus par l'enregistrement des activités alimentaires. 
Par rapport à la ration témoin, l'effet de la ration complète peut être dû à différents facteurs, en général confondus. C'est pourquoi nous avons tenté de les distinguer selon les essais : effet de coupe de la remorque mélangeuse (essai III), effet du niveau d'apport de concentré (essai II et III), effet des différences individuelles entre animaux (essai I), effet de la cinétique d'apport de l'aliment concentré au cours de temps (essai IV).

\section{MATÉRIEL ET MÉTHODES}

Les essais ont été réalisés sur le domaine INRA d'Orcival, non équipé pour faire des études de digestion, durant les hivers 19891990, 1990-1991 et 1991-1992, sur des vaches de race Pie noire (type Holstein) en milieu de lactation. Tous les animaux utilisés ont été alimentés à volonté.

Le schéma général des essais I, II et IV a été le même: après leur vêlage en octobrenovembre, les vaches ont, chaque année, été conduites de la même façon jusqu'en janvier. Une période pré-expérimentale de 2 sem avec mesure des quantités ingérées et des productions laitières a permis ensuite de bien connaître les caractéristiques des animaux sur un même régime pour les apparier. La période de transition a été courte, une semaine avant la période expérimentale qui a toujours duré $11 \mathrm{sem}$.

Chaque année, on a préparé un ensilage d'herbe récoltée au $1^{\mathrm{er}}$ cycle, hâchée finement et additionnée d'un conservateur acide formique-formol à raison d'environ $4 \mathrm{l} / \mathrm{t}$ (ensilage ressuyé la première année, ensilages directs ensuite). Les rations complètes ont été préparées pour chaque lot expérimental avec une remorque mélangeuse Seko de $7 \mathrm{~m}^{3}$ munie de couteaux.

Un temps fixe de $10 \mathrm{~min}$ a été retenu pour préparer toutes les rations complètes.

\section{Essai I}

Dans cet essai, 20 vachès multipares ont été utilisées. Les animaux, après une période pré- expérimentale sur un régime ensilage de maïs + ensilage d'herbe + concentrés, en ration complète, ont été séparés en 2 lots, 8 animaux dans le lot témoin (1) et 12 dans le lot avec la ration complète expérimentale (2). Notre objectif, en mettant 12 vaches dans ce lot, était de pouvoir calculer la régression entre les quantités ingérées de la ration complète et les caractéristiques des animaux, pour savoir comment ils s'adaptaient en fonction de leurs besoins (INRA, 1988). L'apport d'aliment concentré a été calculé pour chaque semaine et chaque animal en tenant compte des besoins des animaux et des quantités d'ensilage ingérées en période préexpérimentale, puis d'une décroissance théorique hebdomadaire de $2 \%$ pour la production de lait. Le lot (2) a reçu une ration complète composée des mêmes aliments que ceux du lot (1), avec une quantité de concentré prévue par animal en moyenne équivalente à celle reçue pour le lot témoin.

L'essai a débuté le 5 mars 1990 et s'est terminé le 20 mai, les animaux étant placés en stabulation entravée sans paille.

\section{Essai II}

Dans cet essai, 27 animaux ont été utilisés (12 primipares) pour constituer 3 lots :

- un lot témoin (1) alimenté séparément avec l'ensilage et l'aliment concentré : pendant les 11 sem de la période expérimentale, les animaux de ce lot ont reçu chaque jour une quantité d'aliment concentré constante, mais différente d'un animal à l'autre pour tenir compte de ses besoins ;

- un lot expérimental (2) avec la ration complète, l'apport de concentré étant calculé pour que les lots 1 et 2 en reçoivent, en moyenne, une même quantité chaque semaine ;

- un lot expérimental (3) avec la ration complète, mais une quantité réduite d'aliment concentré : en effet, les résultats de l'essai I nous ayant montré une ingestion très forte de fourrage, nous avons cherché à diminuer l'apport de concentré pour réduire le coût de la ration.

L'essai a débuté le 4 mars 1991 et s'est terminé le 19 mai. Les animaux étaient en stabulation libre sur paille avec accès à des auges munies de cornadis électroniques. 


\section{Essai III}

En même temps que l'essai II, avec les mêmes aliments, un essai parallèle a été conduit avec 6 vaches en stabulation entravée sans paille pour analyser l'effet de 2 facteurs de variation des quantités ingérées étudiées par ailleurs :

- le hachage de l'ensilage par la remorque mélangeuse (traitements $A$ et $B$ );

- la proportion de concentré dans la ration complète (traitements $C$ et $D$ ).

Pour le traitement $A$, la ration était constituée de l'ensilage normal et le concentré était distribué séparément. Pour le traitement $B$, l'ensilage était haché $10 \mathrm{~min}$ par la remorque, le concentré étant toujours distribué séparément.

Les traitements $C$ et $D$ étaient des rations complètes contenant les mêmes quantités moyennes de concentré par vache que les rations des lots 2 et 3 de l'essai II.

La comparaison des traitements $A$ et $B$ a été faite du 18 février au 31 mars et celle des traitements $C$ et $D$ du $1^{\text {er }}$ avril au 12 mai. Le schéma expérimental était, chaque fois, un carré latin (2 périodes, 3 animaux par traitement) avec 2 sem d'adaptation et 1 sem de mesure.

\section{Essai IV}

Dans cet essai, 16 vaches dont 8 primipares ont été utilisées et regroupées en 2 lots. L'objectif était de comparer la ration classique (ensilage et concentré non mélangés) (lot 1), dont la quantité de concentré était ajustée à l'avance chaque semaine (cf essai l), à la ration complète (lot 2), dont la quantité de concentré était ajustée une fois pour toutes en début d'essai et constante, la quantité totale apportée devant être plus faible que dans le lot témoin pour bénéficier de l'ingestion plus élevée de fourrage attendue avec la ration complète et en faisant l'hypothèse que les productions laitières seraient identiques.

L'expérience a duré du 24 février 1992 au 10 mai, les animaux étant placés en stabulation entravée sans paille. Pour ne pas avoir de déficit en matières azotées de bonne qualité, tous les animaux ont reçu $300 \mathrm{~g} / \mathrm{j}$ de farine de poisson, comptabilisée dans l'aliment concentré.

\section{Caractéristiques des animaux}

Les caractéristiques des animaux en lot sont données dans le tableau I. En moyenne, ces animaux pesaient $631 \mathrm{~kg}$ et produisaient $28,8 \mathrm{~kg}$ de lait à $4 \%$ de $M G$.

\section{Caractéristiques des aliments et distribution}

Les caractéristiques des ensilages utilisés sont données dans le tableau II. L'aliment concentré utilisé a été un concentré d'équilibre constitué d'un mélange de céréales, de pulpe de bette-

Tableau I. Caractéristiques des animaux à la mise en lot.

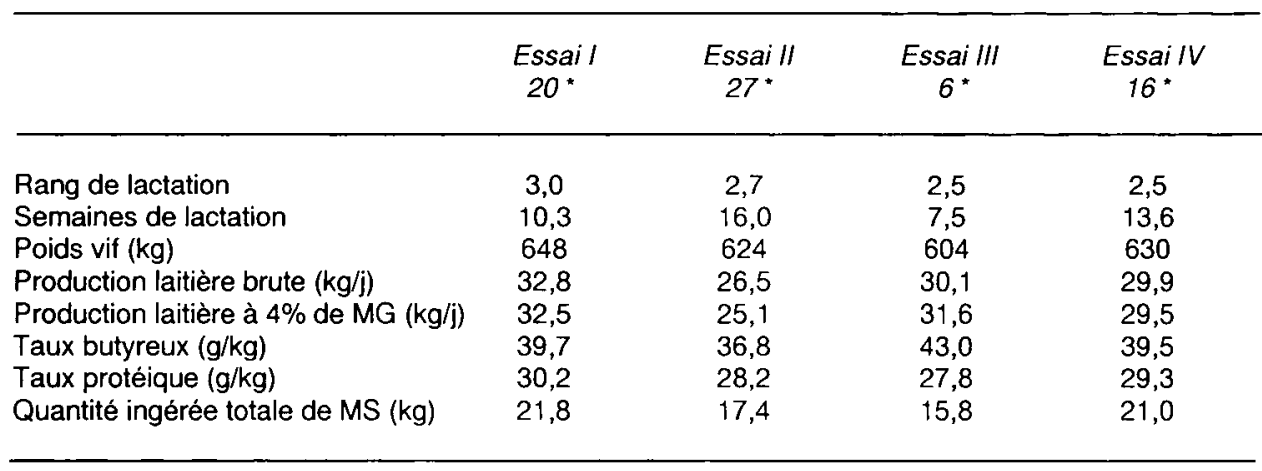

* Nombre d'animaux par essai. 
Tableau II. Caractéristiques des ensilages utilisés.

\begin{tabular}{lccc}
\hline & $\begin{array}{c}\text { Essail: } \\
\text { dactyle }\end{array}$ & $\begin{array}{c}\text { Essais IIIII: } \\
\text { dactyle }\end{array}$ & $\begin{array}{c}\text { Essai IV: } \\
\text { prairie naturelle }\end{array}$ \\
\hline Teneur en MS (\%) & 28,2 & 18,7 & 22,6 \\
Cendres (g/kg MS) & 93 & 80 & 84 \\
Matières azotées totales (g/kg MS) & 163 & 156 & 151 \\
Cellulose brute (g/kg MS) & 263 & 315 & 299 \\
$\mathrm{pH}$ & 3,92 & 4,57 & 3,80 \\
$\mathrm{~N}-\mathrm{NH}_{3}(\% \mathrm{~N}$ total) & 6,3 & 14,2 & 6,5 \\
$\mathrm{~N} \mathrm{soluble} \mathrm{( \%} \mathrm{N} \mathrm{total)}$ & 50 & 61 & 49 \\
Acides (g/kg MS) & & & \\
Lactique & 60 & 2 & 56 \\
Acétique & 14 & 56 & 19 \\
Butyrique & 0,7 & 2,1 & 0,8 \\
Alcools (g/kg MS) & 4,9 & 38,0 & 11,6 \\
Digestibilite de la MO\% (mesure sur moutons) & 74,0 & 68,5 & 66,0 \\
& & & \\
\hline
\end{tabular}

raves et de tourteau $(1,05 \mathrm{UFL} / \mathrm{kg} \mathrm{MS}, 125 \mathrm{~g}$ de PDIN et de PDIE ; $85 \mathrm{~g}$ de cendres, $181 \mathrm{~g}$ de MAT et $100 \mathrm{~g}$ de $\mathrm{CB} / \mathrm{kg}$ de MS). La teneur en MAT de la farine de poisson était de $706 \mathrm{~g} / \mathrm{kg}$ de MS (essai IV). La composition des rations complètes est donnée dans le tableau III.

Les ensilages et les rations complètes ont toujours été distribués en une seule fois le matin entre $9 \mathrm{~h}$ et $9 \mathrm{~h} 30$. Le concentré a été donné à l'auge, la moitié juste après la distribution de l'ensilage, l'autre moitié à $16 \mathrm{~h}$. Dans tous les cas les refus de fourrage (ou de ration complète) étaient enlevés à $8 \mathrm{~h}$.

Par ailleurs, tous les animaux ont reçu des vitamines et des minéraux pour couvrir leurs besoins.

\section{Mesures et interprétation des résultats}

Les quantités ingérées ont été déterminées individuellement par pesée des quantités offertes et

Tableau III. Composition des rations complètes.

\begin{tabular}{lcccccc}
\hline & Essail & \multicolumn{2}{c}{ Essaill } & \multicolumn{2}{c}{ Essai III } & Essai IV \\
& Lot 2 & Lot 2 & Lot 3 & Lot H & Lot B & Lot 2 \\
\hline Teneur en MS (\%) & 34,7 & 24,2 & 21,9 & 24,0 & 21,9 & 28,0 \\
Cendres (g/kg MS) & 91 & 81 & 81 & 81 & 81 & 84 \\
MAT (g/kg MS) & 168 & 163 & 160 & 163 & 161 & 167 \\
CB (g/kg MS) & 218 & 253 & 276 & 254 & 275 & 248 \\
\hline
\end{tabular}


refusées durant 4 j par semaine, le concentré distribué étant pesé tous les jours. Le lait produit a été pesé tous les jours et sa composition a été analysée $2 \mathrm{j}$ par semaine. Les animaux ont été pesés toutes les 2 à 4 sem. ves :

Quelques incidents sanitaires ont été obser-

- 1 indigestion, 1 boiterie et 8 mammites dans l'essai l;

- 1 panaris, 4 mammites dans l'essai II;

- 6 mammites dans l'essai IV.

On a considéré que ces incidents étaient indépendants des traitements étudiés et on a corrigé les baisses temporaires de production laitière qu'ils ont entraînées. Cela s'est traduit par une augmentation de 0 à $0,6 \mathrm{~kg}$ de lait par jour pour chaque lot, ce qui est très faible.

Dans les essais I, II et IV, les mesures retenues sont celles des $2 \mathrm{sem}$ de pré-expérience (covariables) et des 11 sem expérimentales (variables). Elles ont été analysées avec un programme de variance-covariance (logiciel SAS, 1985). Les moyennes ont été ajustées pour tenir compte des différences entre lots lors des périodes pré-expérimentales et donc disposer de résultats directement comparables dans le tableau IV.

Dans l'essai III, seules ont été retenues les mesures faites lors de la semaine 3 de chaque période. Dans cet essai, en plus des mesures habituelles, les durées journalières de mastication des animaux ont été enregistrées (Brun et al, 1984).

\section{RÉSULTATS}

\section{Quantités ingérées}

Dans les essais de longue durée (essai I, II et IV), les animaux ont ingéré en moyenne $12,6 \mathrm{~kg}$ de MS d'ensilage et $6,1 \mathrm{~kg}$ de concentré. La distribution de la ration complète a fait augmenter les quantités ingérées de MS totales de $1,8 \mathrm{~kg}$ en moyenne (lots 2 comparés aux lots 1 ), et ce d'autant plus que l'apport d'aliment concentré était maintenu équivalent entre les 2 types de rations: $+3,6 \mathrm{~kg}$ de MS dans l'essai I, +1,4 kg dans l'essai II et $+0,4 \mathrm{~kg}$ dans l'essai III, pour des apports respectifs de concentrés différents de $+0,2,-0,4$ et $-1,3 \mathrm{~kg}$ de MS (tableau IV).

Les animaux du lot 3 de l'essai II, qui avaient été fortement restreints en concentré, n'ont en revanche pas réussi à totalement compenser: baisse des quantités ingérées totales de $0,26 \mathrm{~kg}$ de MS pour une baisse de concentré de $2,6 \mathrm{~kg}$.

Pour les 12 animaux du lot 2 de l'essai I, la régression entre les quantités ingérées totales QIT et les caractéristiques des animaux (PL4 $=$ production de lait à $4 \%$ de $\mathrm{MG}$; $\mathrm{PV}=$ poids vif en $\mathrm{kg}$ ) a été la suivante :

QIT $=-1,2+0,335$ PL4 + 0,0207 PV $\pm 2,04$ avec $R^{2}=0,60$

Le hachage de l'ensilage par la remorque mélangeuse a augmenté les quantités d'ensilage ingérées de 10\%, dans l'essai III (tableau V).

Enfin, les animaux recevant les rations $C$ et $D$ de l'essai III n'ont pas compensé du tout la baisse d'apport de concentré qui leur était imposée par une augmentation des quantités d'ensilage ingérées.

\section{Durée de mastication des rations (essai III)}

Le fait de remplacer l'ensilage normal par l'ensilage rehaché a fait baisser, non significativement, la durée de mastication de la ration de $3,7 \%$, ce qui est faible, malgré une augmentation des quantités totales ingérées. En revanche, l'ingestion du traitement $D$ a entraîné une forte baisse de la durée journalière de mastication $(-12,4 \%)$, mais avec une baisse importante de la quantité ingérée totale.

$\mathrm{Si}$ on suppose que chaque $\mathrm{kg}$ de MS de concentré nécessite $10 \mathrm{~min}$ environ de 
Tableau IV. Résultats des essais de longue durée (moyennes ajustées par essai).

\begin{tabular}{|c|c|c|c|c|c|c|c|c|c|c|}
\hline \multirow[b]{2}{*}{ Lot } & \multicolumn{3}{|c|}{ Essail } & \multicolumn{4}{|c|}{ Essaill } & \multicolumn{3}{|c|}{ EssailV } \\
\hline & 1 & 2 & ETR & 1 & 2 & 3 & ETR & 1 & 2 & ETR \\
\hline Effectif & 8 & 12 & & 8 & 9 & 9 & & 8 & 8 & \\
\hline \multicolumn{11}{|l|}{$\begin{array}{l}\text { Quantités ingérées } \\
\text { (kg MS/i) }\end{array}$} \\
\hline Ensilage d'herbe & $12,92^{\mathrm{a}}$ & $16,28^{b}$ & 1,59 & $11,6^{\mathrm{a}}$ & $12,96^{\mathrm{a}}$ & $13,48^{b}$ & 0,86 & $13,64^{a}$ & $15,31^{b}$ & 1,06 \\
\hline Concentrés & 6,00 & 6,22 & - & 5,6 & 5,24 & 3,02 & - & 6,60 & 5,31 & - \\
\hline Total & 18,92 & 22,50 & - & 16,76 & 18,20 & 16,50 & - & 20,24 & 20,62 & - \\
\hline \multicolumn{11}{|l|}{ Production par jour } \\
\hline Lait brut $(\mathrm{kg})$ & $27,4^{\mathrm{a}}$ & $28,5^{a}$ & 1,48 & $22,6^{\mathrm{a}}$ & $21,9^{a}$ & $19,9^{b}$ & 1,25 & $26,1^{\mathrm{a}}$ & $25,5^{\mathrm{a}}$ & 1,21 \\
\hline Lait à $4 \%$ MG (kg) & $27,8^{a}$ & $28,0^{a}$ & 1,69 & $21,7^{a}$ & $20,4^{a}$ & $18,9^{b}$ & 1,35 & $25,7^{a}$ & $25,4^{a}$ & 0,94 \\
\hline Matières grasses $(\mathrm{g})$ & $1120^{a}$ & $1109^{a}$ & 83 & $846^{a}$ & $768^{b}$ & $730^{\mathrm{b}}$ & 63 & $1015^{a}$ & $1011^{a}$ & 54 \\
\hline Mat protéiques $(g)$ & $854^{a}$ & $893^{a}$ & 42 & $645^{a}$ & $606^{b}$ & $548^{c}$ & 40 & $781^{a}$ & $764^{a}$ & 36 \\
\hline \multicolumn{11}{|l|}{ Composition du lait } \\
\hline Taux butyreux $(\mathrm{g} / \mathrm{kg})$ & $40,6^{\mathrm{a}}$ & $39,4^{\mathrm{a}}$ & 1,78 & $37,5^{\mathrm{a}}$ & $35,1^{\mathrm{a}}$ & $36,4^{\mathrm{a}}$ & 1,92 & $39,3^{a}$ & $40,0^{\mathrm{a}}$ & 2,65 \\
\hline Taux protéique $(\mathrm{g} / \mathrm{kg})$ & $31,2^{\mathrm{a}}$ & $31,5^{\mathrm{a}}$ & 1,07 & $28,7^{a}$ & $27,6^{b}$ & $27,5^{\mathrm{b}}$ & 0,85 & $30,2^{a}$ & $30,3^{a}$ & 1,04 \\
\hline Cellules $(1000 / \mathrm{ml})$ & $184^{a}$ & $242^{a}$ & 106 & $248^{a}$ & $35 t^{\circ}$ & $351^{\mathrm{b}}$ & 97 & $77^{\mathrm{a}}$ & $96^{a}$ & 42 \\
\hline Poids vif (kg) & $655^{a}$ & $665^{a}$ & 15 & $620^{a}$ & $633^{a}$ & $620^{a}$ & 15 & $641^{a}$ & $637^{a}$ & 16 \\
\hline Gain de poids vif* $(g / j)$ & $+13^{a}$ & $+119^{a}$ & 225 & $-97^{a}$ & $+2^{a}$ & $-370^{b}$ & 237 & $+284^{a}$ & $+67^{a}$ & 205 \\
\hline
\end{tabular}

* Corrigé pour tenir compte des différences de contenus digestifs, considérées comme proportionnelles aux différences de quantités de MS ingérées. Dans un même essai, sur une méme ligne, les valeurs suivies de lettres différentes sont significativement différentes.

mastication (Sauvant et al, 1990), il est possible d'estimer la durée de mastication par $\mathrm{kg}$ de MS de fourrage (tableau V). Ainsi après passage dans la remorque cette durée a baissé de $13 \%$. De même, la diminution du concentré dans la ration complète a fait théoriquement baisser de nouveau de $6,5 \%$ cette durée.

\section{Production et composition du lait}

Dans les essais de longue durée, on laissera de côté le lot 3 de l'essai II qui a été nettement sous-nourri. Dans ces conditions, le passage à la ration complète (lots
2 comparés à lots 1) n'a pas modifié la production de lait brut. La production de lait à $4 \%$ de MG a baissé en moyenne de $0,5 \mathrm{~kg} / \mathrm{j}$, mais non significativement. En effet, le taux butyreux a baissé de 1 point, bien que cette baisse ne soit pas non plus significative. En revanche, le taux protéique n'a pas été modifié.

Dans les mêmes comparaisons, la production de matières grasses par jour a baissé de $31 \mathrm{~g}(-3 \%)$ et celle des matières protéiques de $6 \mathrm{~g}$ seulement, aucune différence n'étant significative. Par ailleurs, le nombre de cellules par $\mathrm{ml}$ de lait a augmenté légèrement, passant de 170 à $230 x$ $10^{3}$, augmentation non significative. 
Tableau V. Résultats de l'essai III.

\begin{tabular}{|c|c|c|c|c|}
\hline \multirow{3}{*}{ Traitement } & \multicolumn{2}{|c|}{$\begin{array}{l}\text { Première comparaison } \\
\text { concentré à part (même proportion } \\
\text { dans les } 2 \text { régimes) }\end{array}$} & \multicolumn{2}{|c|}{$\begin{array}{l}\text { Seconde comparaison } \\
\text { concentré mélangé dans } \\
\text { la remorqueuse mélangeuse }\end{array}$} \\
\hline & Sans hachage & $\begin{array}{l}\text { Haché par remorque } \\
\text { mélangeuse }\end{array}$ & & \\
\hline & $A$ & $B$ & $C$ & $D$ \\
\hline \multicolumn{5}{|c|}{ Quantités de MS ingérées $(\mathrm{kg})$} \\
\hline Ensilage & $8,03^{a}$ & $8,85^{a}$ & $12,60^{a}$ & $12,04^{a}$ \\
\hline Concentrés & 7,87 & 8,35 & 5,00 & 2,80 \\
\hline Total & 15,9 & 17,2 & 17,6 & 14,8 \\
\hline $\begin{array}{l}\text { Production de lait à } 4 \% \\
\text { de } M G(\mathrm{~kg})\end{array}$ & $27,1^{\mathrm{a}}$ & $27,5^{\mathrm{a}}$ & $22,8^{a}$ & $22,3^{a}$ \\
\hline $\begin{array}{l}\text { Durée journalière de } \\
\text { mastication (min) }\end{array}$ & $811^{a}$ & $781^{\mathrm{a}}$ & $1015^{a}$ & $889^{a}$ \\
\hline $\begin{array}{l}\text { Durée de mastication } \\
\text { par kg MS ensilage (*) }\end{array}$ & $91^{a}$ & $79^{b}$ & $77^{a}$ & $72^{\mathrm{a}}$ \\
\hline
\end{tabular}

- En prenant $10 \mathrm{~min} / \mathrm{kg}$ MS concentré (Sauvant et al, 1990).

\section{Bilans et valeur énergétique des fourrages (essais I, II et IV)}

Les animaux ont repris en moyenne $67 \mathrm{~g} / \mathrm{j}$ de poids vif (corrigé pour les variations de quantités ingérées) dans les lots témoins et 63 dans les lots 2 . On constate cependant que le gain de poids vif a augmenté, bien que non significativement, lorsque l'apport de concentré a été équivalent entre les lots 1 et 2 . En revanche; il a baissé dès que l'apport de concentré a été réduit de façon notable.

Compte tenu de ces modifications du poids vif, la valeur énergétique estimée des ensilages à travers les performances des animaux des lots 1 a été de 0,85 UFU $\mathrm{kg}$ de MS (respectivement 0,91-0,76 et 0,87 ). Cette valeur est passée à 0,77 UFL dans les rations complètes, en supposant que la valeur du concentré n'a pas varié.

Les bilans en PDI ont toujours été positifs $(+13 \%$ par rapport aux besoins recommandés) et parfois largement. II n'y a donc pas eu, en principe, de déficit à ce niveau.

\section{DISCUSSION ET CONCLUSION}

Des résultats identiques à ceux des essais déjà publiés et synthétisés entre autres par Hoden et Giger (1984) ont été trouvés, en particulier une ingestion plus élevée avec les rations complètes, des performances comparables, et une tendance à une meilleure reprise de poids si on ne réduit pas l'apport de concentré par rapport à la ration classique. 
Les résultats de l'essai I correspondent à ce schéma, mais ils sont particuliers, compte tenu d'une augmentation considérable des quantités ingérées sans modification de la production laitière. Cette augmentation a pu être facilitée par le fait que l'ensilage était d'excellente qualité, avec une teneur en MS élevée mais elle a entraîné, à coup sûr, une très faible efficacité de l'énergie ingérée, sans qu'une explication correcte puisse être apportée.

L'effet de la remorque mélangeuse sur les quantités ingérées totales a donc été considérable $(+9,6 \%$ entre les lots 1 et 2$)$. Deux causes peuvent être avancées : l'effet du rehachage du fourrage et l'effet du mélange entre le fourrage et l'aliment concentré. Au niveau de l'ensilage, les quantités ingérées ont été augmentées de $18 \%$, ce qui est considérable. Aux 2 causes précédentes s'ajoute une troisième : la diminution de $500 \mathrm{~g}$ de MS de l'apport d'aliment concentré.

D'après l'essai III, l'effet du rehachage par la remorque pourrait expliquer près de la moitié de l'augmentation de l'ingestion d'ensilage. II est probable que l'effet de la diminution du concentré soit négligeable (+0,3 kg de MS si on prend un taux de substitution de 0,6; Dulphy et al, 1987). L'effet du mélange est alors important, mais conforme aux conclusions de la littérature.

Compte tenu de cette augmentation des quantités ingérées totales, nous avons cherché, dans 2 comparaisons, à distribuer moins de concentré avec la ration complète. A priori cela doit être possible s'il y a une substitution fourrage/concentré élevée, donc si le fourrage ingéré est de bonne qualité (Faverdin et al, 1991). C'est ce qui a été vérifié dans l'essai IV avec un très bon ensilage. Dans l'essai II, en revanche, avec un ensilage de mauvaise qualité (moins digestible et mal consommé), cela n'a pas été possible. Les résul- tats de la seconde comparaison de l'essai III ont même été catastrophiques.

En définitive, d'après nos essais, mais il faudrait le confirmer, on devrait pouvoir abaisser l'apport de concentré d'environ $500 \mathrm{~g} / \mathrm{j}$ et par vache, avec une ration complète telle que nous l'avons préparée, sans baisse des performances, si l'ensilage est de bonne qualité, mais non si l'ensilage est de mauvaise qualité. Ce point mériterait d'être vérifié dans la mesure où un handicap de la ration complète préparée mécaniquement est son coût (amortissement du matériel, ingestion des fourrages plus élevée).

Les résultats obtenus peuvent être traduits en termes de valeurs d'encombrement (Dulphy et al, 1987; Faverdin et al, 1987). Ainsi, la valeur d'encombrement moyenne des ensilages distribués séparément de l'aliment concentré a été de 1,06 UEL/kg de MS (respectivement 1,07, 1,12 et 1,00 selon les années; calcul à partir de la capacité d'ingestion théorique des animaux). Après introduction dans les rations complètes et en supposant égale à 0,6 la valeur d'encombrement des concentrés, la valeur d'encombrement moyenne des ensilages est passée à 0,98 , soit une baisse de $7,5 \%$ (lots 2 comparés aux lots 1 ). Pour les rations, la valeur d'encombrement est passée, en moyenne, de 0,92 à $0,87 \mathrm{UEL} /$ $\mathrm{kg}$ de MS $(-5,4 \%)$.

Grâce à la valeur énergétique relativement élevée des rations complètes, les animaux ont de bonnes possibilités de réguler leur ingestion en fonction de leurs besoins. Nous avons essayé de le mesurer dans l'essai I, mais il est évident que le nombre de 12 animaux utilisés était insuffisant. En fait, ces animaux ont mieux ajusté leur ingestion par rapport à leur poids vif qu'à leur production de lait. II aurait fallu une régression d'environ 0,5 entre les quantités ingérées totales et la production de lait. En effet, $1 \mathrm{~kg}$ de lait équivaut à 
0,44 UFL et la valeur réelle de chaque $\mathrm{kg}$ de matière sèche totale ingérée était de 0,88 UFL.

Les résultats de durée de mastication ont été utilisés par ailleurs (Dulphy et al, 1993) et ne seront pas discutés ici. Disons simplement que la durée de mastication du fourrage utilisé par $\mathrm{kg}$ de MS a été élevée, en cohérence avec sa faible digestibilité et sa teneur en cellulose élevée. L'effet du rehachage a été conséquent et celui de la baisse de concentré plus élevé qu'à l'ordinaire.

Comme Hoden et Giger (1984) l'avaient noté, le fait de passer à la ration complète a peu influencé les performances laitières des animaux. Lorsque l'apport de concentré a été maintenu à un niveau comparable (essais I et II), les animaux ont en revanche eu tendance à reprendre un peu de poids.

Une des justifications avancées pour utiliser la ration complète est la meilleure efficacité de sa digestion grâce à l'apport régulier du concentré tout au long de la journée, ce qui réduit les risques d'acidose (Gill, 1979) et les interactions digestives négatives. Cependant, la baisse nette de la valeur énergétique calculée à travers les performances des animaux montre qu'il existe un mécanisme inverse qui s'oppose à l'amélioration de la valeur nutritive de la ration après mélange. Ce mécanisme pourrait bien être un transit plus rapide lié à un niveau d'ingestion plus élevé, et amplifié encore, dans nos essais, par le rehachage du fourrage. II sera intéressant de le vérifier par ailleurs. II y a en effet là un problème que n'ont pas relevé les auteurs des revues que nous avons citées, et qui pourrait être lié au type de matériel utilisé pour préparer la ration complète. II faut noter cependant que la ration complète a été surtout développée pour pouvoir distribuer des quantités élevées d'aliments concentrés aux vaches laitières et de sous-produits peu coûteux. Dans ces conditions, il n'est pas sûr que la valeur énergétique de la ration soit affectée et, même si elle l'est, cela n'a probablement pas de répercussions économiques, la ration complète permettant de faire ingérer des aliments qui ne peuvent pas l'être autrement.

En conclusion, nos essais confirment l'effet positif du passage à la ration complète sur les quantités de MS ingérées par des vaches laitières. Cet effet est amplifié par le rehachage du fourrage dans la remorque, qui permet la préparation d'une telle ration. II devrait permettre de diminuer légèrement l'apport de concentré aux animaux. Cependant, avec des fourrages de bonne qualité et des apports de concentré représentant environ $30 \%$ de la matière sèche de la ration, le recours à la ration complète devra être mûrement réfléchi. En effet, malgré des avantages certains avec ce type de ration, le risque d'observer une baisse de la valeur énergétique, parfois importante, des aliments distribués pose problème. C'est pourquoi des travaux complémentaires sont nécessaires, à la fois pour comprendre les mécanismes qui expliquent la baisse de la valeur énergétique, et si possible les réduire, et pour situer le coût des rations ainsi préparées par rapport à d'autres, voire pour comparer le matériel utilisé dans ces essais à d'autres.

\section{RÉFÉRENCES}

Augeard P, Bazin S, Chenais F, Girard P, Weiss $P$ (1986) Ration complète ou distribution individuelle de concentrès? Bilan de trois années d'expérimentation aux Trinottières (49). Supplément Élevage à Perspectives Agricoles 105, 26-32

Bines JA (1980) Systems for feeding cows. In: Feeding strategies for dainy cows. Seminar ARC, 160 Great Portland Street, London W1N 6DT, 11-1/11-12 
Brun JP, Prache S, Béchet G (1984) A portable device for eating behavior studies. 5th meeting Eur Grazing Workshop

Dulphy JP, Faverdin P, Micol D, Bocquier $F$ (1987) Révision du système des unités d'encombrement (UE). Bull Tech CRZV Theix 70 , 35-48

Dulphy JP, Rouel J, Jailler M, Sauvant D (1993) Données complémentaires sur les durées de mastication chez des vaches laitieres recevant des rations riches en fourrage: Influence de la nature du fourrage et du niveau d'apport d'aliment concentré. INRA Prod Anim 6 (4), 297-302

Faverdin P, Hoden A, Coulon JB (1987) Recommandations alimentaires pour les vaches laitières. Bull Tech CRZV Theix 70, 133-152

Faverdin P, Dulphy JP, Coulon JB et al (1991) Substitution of roughage by concentrates for dairy cows. Liv Prod Sci 27, 137-156

Gill M (1979) The principles and practise of feeding ruminants on complete diets. Grass Forage Sci 34, 155-161

Hoden A, Giger S (1984) Les rations complètes pour vaches laitières. Revue bibliographique. Bull Tech CRZV Theix 58, 45-50

INRA (1988) Alimentation des bovins, ovins et caprins (R Jarrige, ed). INRA, Paris, $471 p$

Lahr DA, Otterby DE, Johnson DG, Linn JG, Lundquist RG (1983) Effects of moisture content of complete diets on feed intake and milk production by cows. J Dairy Sci 66 , 1891-1900

Nocek JE, Steele RL, Braund DG (1986) Performance of dairy cows fed forage and grain separately versus a total mixed ration. $J$ Dairy Sci 69, 8, 2140-2147

Owen JB (1971) Complete diets for ruminants. Agriculture (London) 78, 331-333

Owen JB (1984) Complete diet feeding for cattle. Liv Prod Sci 11, 269-285

Rickaby CD (1978) A review of the nutritional aspects of complete diets for dairy cows. ADAS Q Rev 29, 51-70

SAS Institute (1985) SAS Users' Guide. SAS Institute Inc, USA

Sauvant D, Dulphy JP, Michalet-Doreau B (1990) Le concept d'indice de fibrosité des aliments des ruminants. Prod Anim 3(5), 309318

Sauvant D, Giger S (1984) Quelles spécifications pour les rations complètes des ruminants laitiers ? Bull Tech CRZV Theix 58, 59. 66

Sheehan W, Lawlor MJ, Morris M (1981) Effects of method of feeding supplementary concentrates on the voluntary intake of silage and performance of beef cattle. Ir J Agric Res 20 , $21-25$ 